\title{
Une perspective anthropologique sur la douleur chronique.
}

\author{
Michael Houseman (EPHE, Paris)
}

(Douleurs 5 (2) : 105-107, 2004)

Il existe une différence de perspective fondamentale entre les approches médicale et anthropologique de la douleur: si la médecine met l'accent sur la douleur en tant que modalité particulière de l'expérience corporelle, l'ethnologie s'interroge davantage sur l'inscription de cette expérience au sein d'un réseau particulier de relations. En d'autres termes, et en durcissant quelque peu le trait, tandis que la médecine aborde la douleur avant tout comme phénomène ressenti, l'ethnologie, explicitement ou implicitement, tend à apprécier la douleur comme quelque chose d'infligé.

Afin d'illustrer la portée de cette divergence, considérons brièvement cette modalité particulière de la souffrance qu'est la « douleur chronique ». D'un point de vue médical centré sur les causes matérielles des dysfonctionnements individuels, la douleur chronique s'impose comme infirmité singulière et hautement problématique. En revanche, d'un point de vue anthropologique, qui s'attache moins aux expériences individuelles qu'aux conditions relationnelles (c'est-à-dire sociales) de ces expériences, elle apparaît davantage comme un cas extrême d'un phénomène ordinaire.

Les ethnologues qui travaillent depuis peu sur la douleur dans les services et les centres médicaux spécialisés (par exemple [1], [2]), se sont surtout penchés sur les qualités perceptives et conceptuelles des "mondes" où vivent des patients souffrant de douleurs chroniques. Il se dégage de ces travaux que l'univers de la douleur chronique peut être caractérisé par trois traits principaux.

Le premier est un dérèglement de la perception ordinaire du temps et de l'espace. Le temps peut se ralentir péniblement, ou au contraire s'accélérer à une trop grande vitesse. Assujettie à de tels effets de dilatation ou de contraction du temps, la personne souffrante appréhende difficilement la durée comme un passage d'intervalles réguliers ; il devient compliqué de prévoir les événements à l'avance. Du côté de la perception de l'espace, interviennent tout d'abord des confusions entre l'intérieur et l'extérieur du corps. Dans l'état d'hypersensibilité auquel mène la douleur chronique, les frontières de l'individu deviennent floues : des stimuli externes (des bruits par exemple) peuvent être ressentis comme des intrusions violentes ou, au contraire, un malaise interne peut être projeté sur des éléments de l'environnement. On observe en même temps une confusion entre la partie et le tout du corps, une difficulté à localiser la douleur, ressentie à la fois comme associée à un certain endroit (là où j'ai mal) et vécue comme un fait total (je suis mal). Dans des dérèglements de ce genre, touchant à des repères fondamentaux de la personne, ce n'est pas seulement la perception mais l'appréhension du soi qui se trouve menacée [3].

Un second trait du monde de la douleur chronique consiste en une coupure radicale entre celui qui souffre et les autres. Elaine Scarry [4] résume la situation de la manière suivante : "La douleur arrive parmi nous sans pouvoir être partagée, à la fois comme ce qui ne peut être nié [par celui qui la ressent] et ce qui ne peut être confirmé [par celui qui ne la ressent pas]" (p.4). En effet, de nombreux auteurs ont souligné l'inadéquation du langage face à la douleur, les effets d'alexithymie et les difficultés de communication qu'elle provoque. Entouré de personnes qui, inévitablement, "ne peuvent pas comprendre", l'individu souffrant est renvoyé à lui-même.

Enfin, le monde de la douleur chronique est animé par une urgence explicative, un besoin impératif d'identifier les causes et les raisons de la souffrance : "La douleur n'invite pas 
à l'interprétation [...], nous rappelle Morris [5], elle semble l'exiger" (p.34). Cette recherche ontologique devient d'autant plus pressante qu'elle est fatalement exacerbée par un questionnement de type existentiel : pourquoi moi ?

L'association de ces trois caractéristiques - dissolution des frontières habituelles du moi, retranchement sur soi et besoin pressant d'explication - aboutit à ce qui représente peut-être la caractéristique première de l'expérience de la douleur chronique, à savoir une tendance au dédoublement. Celui-ci s'opère sur plusieurs registres recouvrant différentes dimensions (psychologique, physiologique, sociologique) : "entre le moi et son existence corporelle" [6] (p.16), mais aussi entre le moi endolori et le moi sans douleur ("l'homme qui souffre a un autre corps et il est un autre homme" [7] (p.783), entre un moi inconscient dont les motivations m'échappent et le moi conscient condamné à en subir les peines, entre le moi privé et le moi qu'exige la compagnie d'autrui, etc. [3]. Dans l'ensemble, ces divers mouvements de dédoublement, engagés afin de se situer par rapport à sa douleur, s'organisent autour de deux stratégies [2], lesquelles correspondent, par ailleurs, à ce que Baszanger [8] identifie comme les deux modèles de la prise en charge médicale actuelle de la douleur chronique ("guérison-technique" versus "guérison-gestion"). L'une consiste en une objectivation du mal : la douleur serait une réalité physique qu'il convient de reconnaître en tant que telle pour pouvoir lui faire face ; il s'agit d'un dysfonctionnement du corps pour lequel il faut rechercher un traitement approprié ou, à défaut, qu'il faut apprendre à accepter. L'autre stratégie consiste à subjectiver la souffrance : la douleur serait un phénomène en partie mental sur lequel le patient peut donc avoir une certaine emprise ; on estime pouvoir maitriser le mal en changeant d'attitude. Cependant, face à une forte douleur qui dure, ni l'une ni l'autre de ces voies ne s'avère véritablement adéquate, car l'individu n'arrive ni à se séparer suffisamment de sa douleur, ni à la contrôler suffisamment. Il en résulte un mouvement de va-et-vient constant et quelque peu désespéré entre ces diverses alternatives : une quête de sens à jamais inassouvie.

A écouter les témoignages de personnes souffrant de douleurs chroniques, on ne peut qu'être impressionné par l'énergie qu'elles investissent dans cette tentative de comprendre leur état, de cerner les causes et les raisons de leurs peines, de préciser la nature de cette relation maléfique qui les accable : celle entre moi et ce " $\mathrm{X}$ " qui est à l'origine de la souffrance. Ainsi il arrive le plus souvent que la douleur elle-même soit spontanément dotée d'intentionnalités, devenant sinon un homoncule tout du moins un adversaire ou interlocuteur éventuel - un "elle" ou "ça" - auquel l'individu se soumet ou non, avec lequel il lutte ou négocie, etc.

On ne doit donc pas s'étonner qu'un aspect central de la quasi-totalité des traitements non biomédicaux de la souffrance consiste en l'identification de cet Autre, c'est-à-dire de l'agent responsable dont l'existence semble présupposée par l'expérience même de la douleur : dieux, ancêtres, esprits, sorciers, etc. En effet, au cours de tout traitement rituel de la souffrance, quelle que soit la tradition concernée, la personne souffrante est amenée à inscrire son expérience malheureuse dans une réseau relationnel impliquant au moins trois types d'agents : l'individu souffrant, l'instance qui l'agresse et le personnage médiateur et donc foncièrement ambigu qu'est le thérapeute. Soulignons tout de suite que dans des traitements de ce genre, la mise en évidence de cette interrelation importe plus qu'une définition précise de divers acteurs ou de leurs modalités d'action. Ainsi, des perplexités qui peuvent subsister quant à la nature exacte des agissements des entités nocives ou des thérapeutes (Qu'est ce qu'un esprit ? Comment opère un sorcier? Que fait exactement un guérisseur ?, etc.) n'entravent en rien l'efficacité de ce type de prise en charge. Car, cette efficacité est avant tout d'ordre existentiel : en instituant une relation convenue entre la personne souffrante et son malheur, cette configuration triangulaire permet au malade d'envisager sa souffrance explicitement en des termes intentionnelles. L'incertitude ontologique quand à l'origine de la douleur se trouve clairement levée à au moins un niveau : j'ai mal parce que quelqu'un me fait 
mal. Du coup, pour la personne souffrante, le retranchement sur soi est contrecarré, les interrogations «Quoi? » et "Pourquoi moi? » trouvent une même explication possible dans l'identification de l'agent responsable (Qui), et les frontières menacées du moi peuvent davantage s'affermir. Dès lors, l'individu atteint, dans sa gestion de la souffrance qu'occasionne sa relation difficile avec cet agent nuisible, est plus à même de mobiliser l'ensemble des modalités interactives qui sont à sa disposition en tant que personne: séduction, menace, conciliation, dissimulation, bluff, etc. En somme, une personnalisation de la douleur qu'impliquent des traitements de ce genre permet à l'individu atteint d'accéder à une expérience enrichie et davantage vivable - disons plus « humaine »-de sa souffrance.

Or, il est intéressant de noter que si ces traitements peuvent fournir de pareilles ressources aux personnes souffrantes, ils comportent en même temps certains désavantages pour les individus soignants. Le statut foncièrement ambivalent de ces derniers en tant que médiateurs entre deux sujets antagonistes, l'un victime (le patient), l'autre agresseur (l'infirmité), les oblige à préciser constamment leur propre position. Il est en effet nullement accidentel qu'au cours des consultations, les thérapeutes traditionnels rappellent souvent à leurs patients qu'ils sont du côté du bien et non du mal.

La biomédecine rechigne à reconnaître à la douleur ou à ses causes un statut d'agent doté d'une intentionnalité propre. De même, elle admet avec difficulté la fragilisation de l'autorité du soignant qu'implique cette reconnaissance. La mise en place d'une configuration triangulaire de type «traditionnel» semble donc, dans une large mesure, exclue d'avance. Dans la meilleure des cas, c'est une relation de type duel, n'impliquant que deux agents (le patient et le soignant) et un non-agent médiateur (l'infirmité), qui y est privilégiée. Il en résulte une situation beaucoup plus instable sur le plan relationnel. Si la personne souffrante œuvre spontanément à envisager sa douleur dans un cadre interactif animé par l'attribution d'intentionnalités à autrui, le seul lien patient-soignant s'avère généralement incapable de fournir une armature suffisante pour permettre à l'individu atteint de se situer clairement visà-vis de son malheur. En conséquence, l'individu sous l'emprise d'une douleur chronique se trouve, en fin de compte, renvoyé à lui-même. D'un côté, il est condamné à chercher continuellement à instaurer une séparation ou division au sein de sa propre personne. De l'autre, il est amené à investir, de façon plus ou moins incontrôlée, le réseau de relations qui l'entoure de spéculations malveillantes: ce sont les membres de sa famille, le personnel soignant, d'autres malades, etc., qui deviennent, aux yeux du patient, autant d'agents qui contribuent à son malheur [2].

D'un point de vue anthropologique, où les émotions-sensations prennent sens surtout en référence à leurs conditions sociales d'émergence, la souffrance est avant tout une expérience de la violence: elle n'est pas simplement ressentie mais infligée. En d'autres termes, la supposition d'une altérité agissante (qu'elle soit visible ou invisible, réelle ou imaginaire) s'impose comme aspect essentiel de l'expérience significative de la douleur. Il serait exagéré d'affirmer que toute douleur est nécessairement ressentie comme infligée. Il convient toutefois de souligner combien une telle interprétation s'impose naturellement à celui qui souffre. Des douleurs passagères ou peu importantes, comme lorsqu'on cogne malencontreusement son orteil contre un caillou, ne provoquent pas, de façon automatique, la supposition d'une instance agissante. Une représentation de la souffrance comme animée par une intentionnalité dépendrait donc de l'intensité, de la durée, du degré de contrôle et, éventuellement, de la répétition de la douleur concernée. Néanmoins, et c'est là le point à ne pas négliger, l'attribution du mal à une altérité supposée représente une virtualité de toute expérience de douleur. Combien d'entre nous ne voient-ils pas sur le coup dans ce malheureux caillou (ou dans son pied, ou dans sa propre personne) un antagoniste virtuel, auquel nous adressons éventuellement des paroles de colère, de reproche ou de défi ? 
Est-il souhaitable que la profession médicale tire davantage parti de la tendance spontanée mais bien réelle qu'ont les individus souffrant de douleurs chroniques de personnaliser leurs souffrances ? Cela reviendrait à favoriser une compréhension de la douleur non plus comme un objet qui médiatise les relations entre l'individu atteint et autrui, mais comme un sujet dont la relation vis-à-vis de la personne souffrante est médiatisée par d'autres sujets. Il n'est pas impossible que pour certains patients au moins, cette stratégie thérapeutique, qui consiste à fournir aux personnes souffrantes davantage de moyens relationnelles pour faire face à leur malheur, puisse s'avérer extrêmement utile. Toutefois, rappelons qu'une telle démarche exigerait du personnel soignant qu'ils tempèrent considérablement leur croyance en une science toute puissante, susceptible de guérir à tout coup (à tout coût), et qu'ils assument plus ouvertement l'ambiguïté de leur position en tant que médiateurs privilégiés de relations nuisibles qui ne sont que partiellement sous leur contrôle. Le jeu en vaut peut-être la chandelle.

\section{Références citées :}

[1] Delvecchio Good, M.-J., P.E. Brodwin, B.J. Good et A. Kleinman, eds. Pain as Human Experience: An Anthropological Perspective. Berkeley: University of California Press 1992.

[2] Jackson, J. Chronic Pain and the Tension between the Body as Subject and Object. In T.J. Csordas (ed.) Embodiment and Experience. The Existential Ground of Culture and Self. Cambridge: Cambridge University Press, 1994:201-228.

[3] Good, B.J. A Body in Pain -- The Making of a World of Chronic Pain. In M.-J. DelVecchio Good, P.E. Brodwin, B.J. Good and A. Kleinman, éds. Pain as Human Experience: An Anthropological Perspective. Berkeley: University of California Press, 1992:29-48

[4] Scarry, E. The Body in Pain. The Making and the Unmaking of the World. Oxford: Oxford University Press 1985.

[5]. Morris, D.M. The Culture of Pain. Berkeley : University of California Press 1991.

[6] Buytendjik, F.J.J. De la douleur. Paris: P.U.F. 1951.

[7] Buytendjik, F.J.J. La douleur vécue. In Encyclopaedia Universalis. Paris: Encyclopaedia Universalis France, 1978:782-785.

[8] Baszanger, I. Théorie, techniques et prise en charge : le cas de deux centres de traitement de la douleur. Techniques et culture $1995 ; 25-26: 263-284$. 